\title{
Measurement of Pain and Related Symptoms in Irritable Bowel Syndrome
}

\author{
Affifa Farrukh ${ }^{1}$ \\ ${ }^{1}$ University Hospitals of Leicester NHS Trust
}

June 9,2020

\begin{abstract}
This paper reviews the tools available to assess outcomes of treatment in irritable bowel syndrome, especially the effect on pain. It critically considers their development, relevance and reliability. Its conclusion is that there are few tools which meet the criteria necessary to place confidence in their validity as appropriate measures of patient outcomes.
\end{abstract}

A critical aspect in designing and evaluating trials of treatment in irritable bowel syndrome (IBS), where the desired outcome is a reduction in patients' experience of pain, is the choice of tool to measure changes in pain severity, its frequency and duration. In order to assess the efficacy of a treatment, it is critical that there are before and after measures. In some conditions, this is relatively easy with clear changes in biomarkers. This is not the case in IBS, where the severity of disease is usually reported by patients and sometimes clinicians under-estimate pain and its consequences. Such discrepancies between general practitioners and patients with IBS were reported by Chassany et al (1). Abdominal pain, straining, disease-related concerns and to a lesser extent myalgias, urgency and bloating have been considered as predictive factors of disease severity as perceived by patients. (2)

Despite the importance of pain to patients with IBS, clear questions have not been developed for use in IBS trials. Terms such as "relief of your IBS symptoms" have been used to measure efficacy, but they are not an objective end-point. (3) Regulatory agencies have been generally dissatisfied with such end-points which generate a simple "Yes" or "No". (4) Lackner et al (5) have shown that although recall of specific symptoms such as worst pain or stool frequency is reasonably accurate, this is not the case for typical pain, which is vulnerable to distortion. As a result, the number of IBS specific tools is limited.

In 1997, Francis et al 6) developed the Irritable Bowel Severity Scoring System (IBS-SSS). It was the first simple method of monitoring the progress of the disease and its treatment. The questionnaire evolved and was simplified over a number of years rather than being developed from formal patient involvement. It has 9 stem questions of which 3 concern abdominal pain or discomfort. Five questions have scores of between 0 and 100 each, based on visual analogue scales. A score below 75 is seen in healthy people or those in remission whilst 75 - 175 indicates mild disease, 175 - 300 moderate disease and over 300 severe disease.

IBS-SSS is frequently used in clinical trials to monitor the progress of the disease and treatment effect. However, there are several concerns regarding it. It is an unwieldy 4 page document and when its readability is scored with on-line software, such as that provided by ReadabilityFormulas.com, the Flesch Reading Ease Score is 64.5 , which is generally considered suitable for a person with an average reading age,(7) whilst the Gunning Fog Index is 11.1 indicating that it would be considered a "Hard Read". (8) Both indices are of American origin and consider sentence length and word complexity, but have been generally accepted as 
useful tools in assessing healthcare literature. (9) Such scores mean that a significant number of people in the UK will have difficulties understanding the text. (10) 2012)

The Irritable Bowel Syndrome Quality of Life (IBS-QOL) instrument measures quality of life. (11) The development of this questionnaire and its translation was been driven by Novartis Pharmaceuticals Corporation and its agents. (12). It is easier to read and faster to complete than the IBS-SSS, with a Flesch Reading Ease Score of 76.6 ("Fairly easy to read") and a Gunning Fog Index of 5.1 ("Easy to read"). It measures psychological well-being, rather than function, and has been translated into several languages. It has 34 items with a 5 - point response scale. Although many of the 34 items could be affected by pain, it is not specifically mentioned.

International concerns about the need to have adequate tools to measure outcomes led to the Food \& Drug Administration issuing Patient Reported Outcomes. (PROs) in 2009. (13) They consider that good PROs and their appropriateness require no intervention or interpretation by clinicians or anyone else. In clinical trials good PROs can be used to measure the effects of an intervention on a single symptom or group of symptoms, or on daily functioning or the severity of a disease. In IBS, it will be necessary to define whether pain relief is the main desired outcome or a global improvement in pain, bloating, defaecation habit and ability to live a normal life are the desired endpoints.

An important aspect in the development of a PRO, which was flagged up in the FDA advice, was the way in which it originated. Patients are crucial. Through mechanisms such as interviews and focus groups PROs can be designed to capture what matters to patients and so are valid. For such reasons, the FDA recommended that when individual scores are added together to give a single overall score it is important that the score represents a recognisable domain rather than being an artificial entity. Kitzinger (14) confirmed the need to ensure that focus groups represented a cross section of society either through use of multiple groups or, less favourably, one group with a widespread membership. However, the Gastrointestinal Pain Pointer, which is an electronic tool developed with the help of patient and control groups representative of Caucasians, African Americans and Asians is the only instrument where this has been attempted. (15) In addition, in a number of studies there has been a failure to recognise that an instrument cannot be simply translated and used in a new culture. Failure to validate the translation means that the result of such a study cannot be relied on.

Based on such advice, Fehnel et al (16) identified incomplete bowel movements, abnormal stool frequency and consistency, and abdominal pain, discomfort, and bloating as common symptoms, but the one feature that concerned patients was abdominal pain. In an attempt to develop reliable PROs Ballou \& Keefer (17) asked patients with IBS to complete an on-line survey. Three factors emerged as important: pain catastrophisation, visceral hypervigilance and extraintestinal hypervigilance. Their tool is patient-completed and has 15 items to cover these areas, but has yet to be used in any clinical trials.

In 2015 Mujajic et al (18) reviewed 110 papers, which had assessed the numerous tools used to assess pain in IBS. They concluded that the most useful questionnaires were:

1. Spiegel et al's 10-point Numeric Rating Scale (2), as it was the best validated tool to measure responses to treatment

2. The IBS - Symptom Severity Scale (IBS-SSS) is the best suited to measure abdominal pain because it has been correlated with physical measures such as the pain induced by rectal distension, is reproducible and is psychometrically robust. (6)

3. Visceral Sensitivity Index (VSI) for measurement of emotional response to abdominal pain, but not to its intensity. (19)

Mujajic et al (18) had identified 7 clinical trials which used Visual Analogue Scales to assess chronic abdominal pain in IBS. However, the authors point out that it has not been psychometrically tested for use in IBS and only measures one dimension of pain intensity. This problem has been overcome by Bengtsson et al (20) who developed a specific and validated Visual Analogue Scale for IBS. The questionnaire has 5 domains with 15 items and was psychometrically sound. 
A Numeric Rating Scale had been used in 4 clinical trials and a 10 point scale used by Spiegel et al (2) was found to have high validity and sensitive clinical responsiveness. It correlated well with IBS-SSS and generic quality of life measures and resource utilisation by patients.

Mujajic et al (18) identified 15 different multi-item questionnaires used in IBS for the assessment of one or more dimensions of abdominal pain. Of these, the VSI is useful for assessing gastrointestinal-specific anxiety, the cognitive, affective, and behavioural response to fear of gastrointestinal sensations, symptoms, and the context in which these visceral sensations and symptoms occur. (19) Its questions reflect the anxieties experienced by many patients with IBS and show that chronic and recurrent abdominal pain can have a devastating effect on patients with IBS sufferers.

\section{Conclusions:}

There are many and varied tools for measuring pain and other aspects of IBS. In practice PROs are likely to best reflect the benefits of any intervention designed to improve patients' experience of pain and its impact on aspects of day-to-day life. There are clear criteria which should be adopted by those setting up clinical trials as to which tests are most reliable, reproducible and valid.

\section{References:}

1. Chassany O, Le-Jeune P, Duracinsky M, Schwalm MS \& Mathieu M (2006) Discrepancies between patient-reported outcomes and clinician-reported outcomes in chronic venous disease, irritable bowel syndrome, and peripheral arterial occlusive disease. Value Health 9 (1): pp $39-46$

2. Spiegel B, Strickland A, Naliboff BD, Mayer EA \& Chang L (2008) Predictors of patient-assessed illness severity in irritable bowel syndrome. American Journal of Gastroenterology103(10): pp 2536 $-2543$

3. Andrae DA, Patrick DL, Drossman DA \& Covington PS (2013) Evaluation of the Irritable Bowel Syndrome Quality of Life (IBS-QOL) questionnaire in diarrheal-predominant irritable bowel syndrome patients. Health \& Quality of Life Outcomes 11: p.208

4. Food \& Drug Administration (2009) Guidance for Industry Patient-Reported Outcome Measures: Use in Medical Product Development to Support Labeling Claimshttps://www.fda.gov/downloads/Drugs/GuidanceComplianceRegulatoryInformation/Guidances/UCM193282.p (Accessed 18/2/2019)

5. Lackner JM, Jaccard J, Keefer l, Firth R, Carosella AM, Sitrin M \& Brenner D (2014) The accuracy of patient-reported measures for GI symptoms: a comparison of real time and retrospective reports.Neurogastroenterology \& Motility 26(12): pp 1802 - 1811

6. Francis CY, Morris J \& Whorwell PJ (1997) The irritable bowel severity scoring system: a simple method of monitoring irritable bowel syndrome and its progress. Alimentary Pharmacology $\& 5$ Therapeutics 11: pp $395-402$

7. Flesch R (1987) The Art of Readable Writing Wiley

8. Gunning R (1952) The Technique of Clear Writing. McGraw Hill

9. Berry D (2006) Health Communication. Theory and Practice McGraw Hill Education (UK)

10. Jama D \& Dugdale G (2012) Literacy: State of the Nation. A Picture of Literacy in the UK Today National Literacy Trust.

11. Patrick DL, Drossman DA, Frederick IO, DiCesare J \& Puder KL (1998) Quality of life in persons with irritable bowel syndrome: development and validation of a new measure. Digestive Diseases and Sciences43(2): pp $400-411$

12. Patrick DL (undated) Information Sheet on the Irritable Bowel Syndrome-Quality of Life Measure (IBS-QOL). http://depts.washington.edu/seaqol/docs/IBS-QOL_Info.pdf (Accessed 20/2/2019)

13. Food \& Drug Administration (2009) Guidance for Industry Patient-Reported Outcome Measures: Use in Medical Product Development to Support Labeling Claimshttps://www.fda.gov/downloads/Drugs/GuidanceComplianceRegulatoryInformation/Guidances/UCM193282.p (Accessed 18/2/2019)

14. Kitzinger J (1995) Introducing focus groups British Medical Journal 311: pp 299 - 302 
15. Henderson WA, Rahim-Williams B, Kim KH, Sherwin LB, Abey SK, Martino AC, Fourie NH, Heitkemper MM \& Zuccolotto AP (2017) The Gastrointestinal Pain Pointer. A valid and innovative method to assess gastrointestinal symptoms. Gastroenterology Nursing 40(5): pp $357-363$

16. Fehnel SE, Ervin CM, Carson RT, Rigoni G, Lackner JM \& Coons SJ (2017) Development of the Diary for Irritable Bowel Syndrome Symptoms to assess treatment benefit in clinical trials: Foundational Qualitative Research. Value in Health 20(4): pp 618 - 626

17. Ballou S \& Keefer L (2017) Development of the irritable bowel syndrome affective scale: a brief selfreport measure for clinical and research settings. European Journal of Gastroenterology $\&$ Hepatology 29(7): pp $849-854$

18. Mujajic Z., Keszthelyi D, Aziz Q, Reinsisch W, Quetglas EG, De Leonardis F, Segerdahl M \& Masclee AAM (2015) Systematic review: instruments to assess abdominal pain in irritable bowel syndrome.Alimentary Pharmacology \&3 Therapeutics 42: pp 1064 - 1081

19. Labus JS, Bolus R, Chang L, Wiklund I, Naesdal J, Mayer EA \& Naliboff BD (2004) The Visceral Sensitivity Index: development and validation of a gastrointestinal symptom-specific anxiety scale.

20. Bengtsson M, Ohlsson B \& Ulander K (2007) Development and psychometric testing of the Visual Analogue Scale for Irritable Bowel Syndrome (VAS-IBS) BMC Gastroenterology 7: p16 